\title{
ESTRATEGIAS ACTIVAS DE APRENDIZAJE PARA CIENCIAS EXACTAS DEL BACHILLERATO GENERAL UNIFICADO (BGU).
}

Dra. Nancy Guadalupe Morales Guevara ${ }^{1}$ Dra. Betty Tacoaman Acurio ${ }^{2}$ Dra. Nancy Piedad Lucero Borja ${ }^{3}$ Lic. Juan Carlos Tobar Segovia ${ }^{4}$

\begin{abstract}
In education there are difficulties for the correct transmission of knowledge, it is felt that within the curriculum develops the application of active strategies for the learning of Exact Sciences in the BGU, since the Ecuadorian teacher is immersed in some sectors within traditional education.

From the experience of teachers in the classroom, active strategies should be employed to solve learning problems, in some cases must be systematically linked to practice with scientific content, which should develop students a sense of research and responsibility, thus generating in them initiatives that develop an active, propositive and innovative learning. The methodology used for the present project is based on bibliographical research with a qualitative-quantitative approach, which will collect the information and must be processed to obtain the statistical tables, which will serve as a starting point for the development and implementation of a guide for the management of active strategies for the area of exact sciences, will allow the development of logical - abstract thinking, through the correct use of the teaching - learning process in a concrete and creative way.
\end{abstract}

\section{Keywords}

Strategies, Activities, Learning, Exact Sciences, Bachelor

\section{CóDIGO UNESCO: Educación 531204}

\section{RESUMEN}

En la educación existen dificultades para la correcta transmisión del conocimiento, se siente que dentro del currículo se desarrolla la aplicación de estrategias activas para el aprendizaje de Ciencias Exactas en el BGU, puesto que el maestro ecuatoriano está inmerso en algunos sectores dentro de la educación tradicional.

\footnotetext{
${ }^{1}$ Unidad Educativa Patate; Ambato, Ecuador; morales2011@gmail.com

${ }^{2}$ Unidad Educativa Pedro Fermín Cevallos; Ambato, Ecuador; btacoaman@yahoo.es

${ }^{3}$ Colegió Pasa; Ambato, Ecuador; pied.bor@gmail.com

${ }^{4}$ Unidad Educativa Bicentenario; Ambato, Ecuador; juancatobarsegovia@hotmail.com
} 
Desde la experiencia de los maestros en el aula, las estrategias activas deben ser empleadas para dar solución a los problemas de aprendizaje, en algunos casos se deben vincular sistemáticamente a la práctica con el contenido científico, que debe desarrollar los estudiantes un sentido de investigación y responsabilidad, logrando así generar en ellos iniciativas que desarrollen un aprendizaje activo, propositivo e innovador. La metodología utilizada para el presente proyecto se basa en la investigación bibliográfica con un enfoque cuali-cuantitativa mediante, la cual recogerá la información y deberá ser procesa para obtener los cuadros estadísticos, los mismos que servirán como punto de partida para el desarrollo e implementación de una guía de manejo de las estrategias activas para el área de ciencias exactas, las mismas permitirán desarrollar el pensamiento lógico - abstracto, mediante el uso correcto del proceso de enseñanza - aprendizaje de manera concreta y creativa.

\section{Palabras clave:}

Estrategias, Actividades, Aprendizaje, Ciencias Exactas, Licenciatura

\section{INTRODUCCIÓN}

\section{DESCRIPCIÓN DETALLADA DEL PROYECTO}

Las estrategias activas son muy importantes en el proceso de enseñanza- aprendizaje, va en función de los objetivos fijados por la institución y asignatura, lo cual requiere tener docentes preparados con una visión capaz de poder brindar a sus estudiantes las mismas oportunidades y condiciones.

Los Objetivos de los docentes y de los estudiantes, es lograr alcanzar un conjunto de variables que lleve a la comprensión de los conocimientos, para obtener un aprendizaje significativo, esto va depender básicamente de la metodología utilizada por el docente para la consecución de objetivos académicos.

Según (Prégent), 1990 nos dice que no existe un método adecuado que va a mejorar el proceso de enseñanza - aprendizaje, tenemos que observar los tipos de objetivos que deseamos cumplir para el desarrollo de un pensamiento crítico y un aprendizaje autónomo, los mismos tienen que ser eficaces, con atributos más a la cantidad y calidad de trabajo personal.

Una afirmación para la enseñanza, es la participación del estudiante específicamente, donde cae la responsabilidad del aprendizaje va a depender directamente de la actividad que realice, donde se pueda generar un aprendizaje más profundo, significativo y duradero, que logren facilitar la transferencia a contextos más heterogéneos.

Un aprendizaje de estas características, en donde se tiene que aplicar varias estrategias activas de enseñanza, demanda que el estudiante logre conseguir obtener sus propias conclusiones a los diferentes problemas planteados, obteniendo resultados satisfactorios y 
sobre todo motivado, con un pensamiento lógico, incentivando en el estudiante el deseo de investigar y sobre todo de aprender a aprender con sentido crítico. (Metodologías Activas para la formación de competencias.) Aparo Fernández March.

Los docentes frente a las estrategias de aprendizaje tienen dificultad en aplicarlas en el aula para generar un nuevo proceso de enseñanza y aprendizaje, el docente debe proponer nuevos procesos activos donde apliquen nuevas estrategias activas, las mismas que genere en el estudiante el pensamiento crítico.

Enseñar mediante adecuadas estrategias metodológicas, se convierte en un proceso en donde están inmersos los docentes para generar cambios en la educación y romper paradigmas que sean capaces de generar crear y desarrollar soluciones de problemas educativos.

Otros de los problemas para implementar estrategias activas es la motivación del estudiante durante el proceso aprendizaje, en cierta manera los modelos tradicionales de enseñanza que el docente utiliza generan desmotivación siendo la consecuencia del bajo rendimiento, convirtiéndose en un estudiante mecánico y memorístico. (Estrategias de Aprendizaje) Luz Amparo Noy Sánchez.

Es muy importante el desarrollo del pensamiento crítico en el estudiante, ya que constituye una de las habilidades mentales esenciales del ser humano para poder desarrollar gran parte de su capacidad de abstracción y razonamiento que se convierten en experiencias individuales y sociales.

Como lo afirma Dewwy (1986) "Los pensamientos y las ideas no pueden ser trasmitida de una mente a otras".

Las metodologías activas se van a concebir, como estrategias facilitadoras y promotoras de un pensamiento crítico, específicamente desde la perspectiva de eficiencia y asertividad, mediante la utilización de mecanismos participativos tanto fuera como dentro del aula , así también mediante la utilización de recursos tecnológicos y bibliográficos .

Las estrategias activas tienen su origen en la pedagogía crítica y en la teoría humanista, en donde se enfocan en la parte integral del ser humano y logran potenciar el desarrollo humano. Mediante la utilización de estas técnicas, existe una predisposición hacia un pensamiento y una actitud inquisitiva, no solo por detallar las diferentes tipos de conceptualizaciones sino por crear y utilizar la lógica dentro de los análisis de los libros, documentos que se logran presentar tanto dentro como fuera del aula, lograr que los estudiantes puedan defender los argumentos.

Es muy importante lograr el pensamiento crítico en cada estudiante, lo cual va a estimular $\mathrm{y}$ fortalecer varios tipos de habilidades cognitivas y actitudinales, de una manera donde 
enseñen a sus estudiantes a pensar críticamente, adquiriendo una metodología propia con un pensamiento investigativo con una percepción racional y lógica. Rosa Inés Lira Valdivia (las metodologías activas).

Los modelos educativos de los países Europeos de Educación tiene una necesidad de enseñar por medio de metodologías activas, dando específicamente al estudiante un protagonismo dentro del proceso de enseñanza Aprendizaje (Durán et al ., 204; Marinez Lirioda, 2007). En este instante del aprendizaje, el docente se va a convertir en diseñador de experiencias intelectuales para los estudiantes (Smith y Mc Grregor, 1992).

El alumnado activo en su aprendizaje, señala Morales Vallejo (2008:22):

"Una enseñanza centrada en el aprendizaje supone para el alumno un papel más activo, un mayor compromiso y responsabilidad por su propio aprendizaje y un enfoque profundo en su estilo de aprendizaje (como, por ejemplo, menos utilización de la memoria en vez de la comprensión)."

Se estables el siguiente objetivo. Implementar estrategias activas de interaprendizaje en las ciencias exáctas del bgu de la unidad educativa municipal del milenio bicentenario, y sus objetivos específicos:

- Identificar los tipos de problemas de aprendizaje de los estudiantes del BGU en las Ciencias Exactas.

- Seleccionar las técnicas de aprendizaje activo adecuadas, a la necesidad cognoscitiva y al desarrollo de las macro destrezas.

- Elaborar una guía de técnicas activas para promover la actualización Docente en el proceso de enseñanza aprendizaje para motivar a los estudiantes.

- Aplicar la guía de técnicas activas a los estudiantes del BGU de la UEMM Bicentenario.

- Evaluar

\section{FUNDAMENTACIÓN CIENTÍFICA TÉCNICA}

Los docentes tienen la responsabilidad, de formar personas altamente preparadas, y con flexibilidad mental para adaptarse a los cambios que ocasiona la introducción de nuevas tecnologías. Estamos en un momento en que se ha perdido la idea de una carrera para toda la vida. De aquí se deriva, la importancia de tener unos conocimientos afianzados que lo suministran las asignaturas de Ciencias Exactas.

Al aplicar técnicas activas se logrará una actitud positiva de los estudiantes hacia las ciencias exactas, la amplia gama de posibilidades que se le ofrece, permitirá activar la inteligencia y la memoria. Las técnicas activas permiten motivar el aprendizaje, mediante la estimulación del interés y la diversión, en el aprendizaje de los conocimientos científicos. 
Ayudará a establecer conexiones entre las ciencias exactas y los fenómenos del mundo real, incentivando al estudiante a promover la interacción con el docente.

\section{METODOLOGÍA}

\section{INVESTIGACIÓN DE CAMPO}

Esta modalidad permitirá realizar el trabajo directo con los estudiantes del BGU de la UEMM Bicentenario en los que se ha visto que presentan problemas de aprendizaje debido al manejo inadecuado de las estrategias activas en cada una de las asignaturas de Ciencias Exacta como Química y Matemáticas

También es una investigación bibliográfica ya que se procedió a la revisión de libros, tesis de tercer nivel, cuarto nivel, tesis doctorales, artículos científicos, páginas web, permitiéndonos ampliar los conocimientos sobre los nuevos paradigmas, teorías, enfoques y modelos educativos, los mismo que permitirán el análisis de una propuesta metodológica.

El proyecto se presenta como la solución al problema del inadecuado manejo de las estrategias activas de enseñanza y del bajo rendimiento de los estudiantes del BGU. Es muy importante desarrollar este proyecto ya que a través de él se podrá ayudar a los docentes y estudiantes de BGU de la UEMM Bicentenario, mediante la elaboración de la guía del manejo creativo de las estrategias activas en las asignaturas de ciencias exactas (Química y Matemáticas)

Así también se desarrollarán metodologías creativas, novedosas mediante la utilización de las TICs, como instrumentos de integración a las aulas y en especial la innovación de los métodos de enseñanza, los mismos que permitirán desarrollar el pensamiento crítico y lógico abstracto en los estudiantes

\section{NIVEL O TIPO DE INVESTIGACIÓN}

Exploratoria: Porque permite identificar el problema mediante el análisis de las características fundamentales del entorno. En la fase inicial nos facilita estudiar, analizar, reconocer, evidenciar, y se describirán las particularidades del problema en el contexto Investigado. Así también el nivel descriptivo el mismo que nos guiarán a establecer de qué manera se presenta el problema y especificar las cualidades que nos permitirán medir y evaluar las dimensiones y componentes del problema.

La necesidad de proporcionar materiales educativos concretos con la finalidad de ayudar a los estudiantes en la activación de conocimientos en el aprendizaje de la Matemática, además para el desarrollo del proyecto educativo se cuenta con los recursos materiales, tecnológicos y económicos que permiten su desarrollo e implementación de manera exitosa. 


\section{RESULTADOS ESPERADOS}

Con el presente proyecto se pretende fomentar el conocimiento de T.A.A., en los establecimientos mediante una capacitación constante de los docentes con la ayuda de una guía que sea fácil de comprender y aplicar sin olvidar que el principal protagonista es el profesor, siendo él quien llevará al estudiante a cumplir con sus expectativas pero con mucha creatividad para adaptar de la mejor manera estas técnicas, y así obtener aprendizajes significativo

\section{FINANCIAMIENTO}

\begin{tabular}{ll}
\hline Recurso a Utilizar en el proyecto & Costo Total \\
\hline Recurso Humanos & 500 \\
Recursos físicos y bibliográficos & 200 \\
Servicios especializados & \\
Total & $\mathbf{7 0 0}$ \\
\hline
\end{tabular}

Tabla $N^{\circ}$ : Recurso financieros

Elaboración: Grupo Investigador

\section{ACTIVIDADES DEL PROYECTO}

- Identificación del Proyecto

- Realización de Encuestas a Docentes

- Realización de Encuestas a estudiantes

- Planificación del Proyecto

\section{SOSTENIBILIDAD}

Dentro del proyecto que se ha puesto en marcha para mejorar las Estrategias activas en la UEMM Bicentenario, ha sido acogido con gran interés por la razón de que propone nuevos cambios dentro del proceso de enseñanza- aprendizaje.

El apoyo por parte de la Universidad Técnica de Ambato, dentro del área del CEPOS los mismos que nos han permitido que nuestro proyecto se pueda difundir mediante el Primer Encuentro Académico, donde los estudiantes van participar y verificar que con las técnicas activas que pueden facilitar el proceso aprendizaje.

La posibilidad de involucrar a otras instituciones existe y es crucial, porque es un proyecto que puede ser implementado en todas las instituciones educativas, pues se proponen 
herramientas para que el aprendizaje sea efectivo los beneficiarios serán los estudiantes quienes lograrán aprendizajes significativos en forma fácil y divertida, de igual forma se beneficiarán los docentes de las asignaturas de Ciencias Exactas que dispondrán de estrategias para hacer el proceso de aprendizaje más activo y acorde con la tecnología actual.

\section{BENEFICIARIOS DEL PROYECTO}

Con el presente proyecto de implementación se beneficiará de manera directa a estudiantes del BGU y docentes de los asignaturas de Ciencias Exactas interrelacionando procesos de enseñanza aprendizaje para fortalecer la didáctica y metodologías activas, las misma que permitirán desarrollar el pensamiento crítico y abstracto, así también emocional, psicomotriz y cognitivo de los estudiantes, haciendo de esta manera amenas las clases y divertidas para aprender a aprender mediante el uso de las TIC`s

Una vez ejecutado nuestro proyecto se tiende a futuro utilizar correctamente las técnicas activas en el aprendizaje de las Ciencias Exactas por parte de los docentes interrelacionados con los estudiantes, para alcanzar los estándares de calidad propuestos en el Plan Decenal.

Proyectos de Investigación Básica.- Se beneficiarán los 32 estudiantes de Cuarto Año de Educación Básica y 6 Docentes del Área de Matemática de la Escuela Isabel La Católica, los mismos que participarán directamente en las actividades del proyecto, mejorando la activación de conocimientos en el Área de Matemática.

Además se pretende motivar al docente hacia la implementación de dichas técnicas activas que se incluirán en las planificaciones y así despertar la responsabilidad de insertar en todas sus clases para diferenciar escalas de aprendizajes, los cuales no solo reflejan cuantitativamente, sino, cualitativamente dándonos cuenta que el estudiante logra interrelacionar los saberes como competencias cognitivas, procedimentales y actitudinales, en una palabra se conviertan en un ser "holístico" como demanda la era científica a la que nos exponemos. Enfocando a los alumnos hacia el paradigma crítico propositivo, contribuyendo al cumplimiento del perfil de salida y objetivos de cada asignatura, creando un ambiente de satisfacción y entrega entre docentes-estudiante.

Estas técnicas van a crear un ambiente de entrega en conformidad a los estilos de aprendizaje, esperando no perfeccionar al joven en una ciencia, más bien abrir su mente y disponerla al cambio según se lo proponga.

Se espera que la institución sea pionera en aplicar correctamente las técnicas activas de aprendizaje y promulgue información a los distritos y circuitos de cada maestro integrante de este trabajo. A diario se detectan problemas de aprendizaje que traen o se crean producto de la escasa utilización de técnicas activas dentro del área de Ciencias Exactas con lo cual se deslinda estudiante - conocimiento, evidenciando promedios poco significativos. 
La guía de técnicas activas se realizará mediante talleres a los maestros donde se seleccionará el manejo y aplicación en cada área, mediante seguimientos periódicos y resultados de notas en cada parcial para establecer conclusiones y recomendaciones. Con la disponibilidad de aumentar guías en pos de mejorar la educación en los márgenes del buen vivir. Para la transferencia de resultados se ha considerado medios como revisión bibliográfica y tesis sobre el tema, para mejorar la calidad de educación, también se ha recopilado ejemplos concretos sobre dichas técnicas y se ha seleccionado las más factibles

\section{CONCLUCIONES}

- Con el presente proyecto de implementación se beneficiará de manera directa a estudiantes del BGU y docentes de los asignaturas de Ciencias Exactas interrelacionando procesos de enseñanza aprendizaje para fortalecer la didáctica y metodologías activas, las misma que permitirán desarrollar el pensamiento crítico y abstracto, así también emocional, psicomotriz y cognitivo de los estudiantes, haciendo de esta manera amenas las clases y divertidas para aprender a aprender mediante el uso de las TIC's

- Una vez ejecutado nuestro proyecto se tiende a futuro utilizar correctamente las estrategias activas en el aprendizaje de las Ciencias Exactas por parte de los docentes interrelacionados con los estudiantes, para alcanzar los estándares de calidad propuestos en el Plan Decenal.

- La guía de estrategias activas se realizará mediante talleres a los maestros donde se explicara el manejo correcto y la aplicación en cada área, mediante seguimientos periódicos y podrán ser evidenciar los resultados en cada parcial para establecer conclusiones y recomendaciones. Con la disponibilidad de aumentar guías en pos de mejorar la educación en los márgenes del buen vivir. Para la transferencia de resultados se ha considerado medios como revisión bibliográfica y tesis sobre el tema, para mejorar la calidad de educación, también se ha recopilado ejemplos concretos sobre dichas estrategias y se ha seleccionado las más factibles.

RECEIVED: SEPTEMBER, 2016

REVISED: DECEMBER, 2016

\section{Bibliografía}

- Valdivia, R. I. L. (2011). Las metodologías activas y el foro presencial: su contribución al desarrollo del pensamiento crítico/Active learning methodologies and syncronous forum: its contribution to the development of critical thinking. Actualidades investigativas en educación, 10(1). 
- Martínez Lirola, M., Català Cobos, P., \& Díaz Soria, M. I. (2013). Aprender colaborando: estrategias de aprendizaje colaborativo integradas en el aula universitaria.

- Restrepo F. (2007). Habilidades investigativas en niños y niñas de 5 a 7 años de instituciones oficiales y privadas de la ciudad de Manizales (tesis de grado). Universidad de Manizale, Manizales, Colombia.

- Moya, M. (2011). La investigación científica y su incidencia en el aprendizaje de los estudiantes de la carrera educación básica de la modalidad semipresencial de la Facultad de Ciencias Humanas y de la Educación de la Universidad Técnica de Ambato (tesis posgrado). Universidad Técnica de Ambato, Ambato, Ecuador.

- Sapiens. (2008). Fácil y divertido: estrategias para la enseñanza de la ciencia en Educación Inicial. Revista Universitaria de Investigación., Año 9, No. 2, pp. 133.

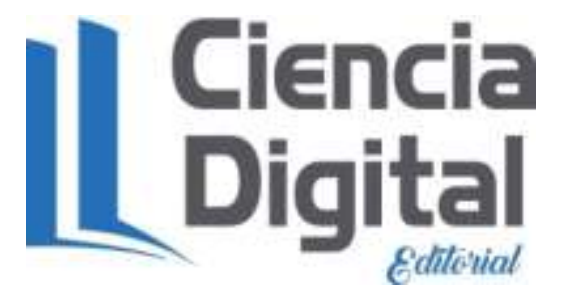

El artículo que se publica es de exclusiva responsabilidad de los autores y no necesariamente reflejan el pensamiento de la Revista Ciencia Digital.

El articulo queda en propiedad de la revista y, por tanto, su publicación parcial y/o total en otro medio tiene que ser autorizado por el director de la Revista Ciencia
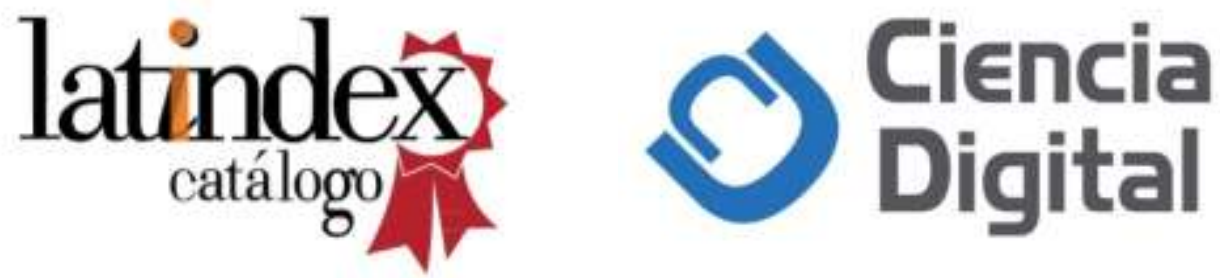